\title{
A Post-Mastectomy Radiation Therapy Dose Distribution Study
}

\author{
Xin Qian1, Kalpana Vaidya', Lindsay Puckett ${ }^{1}$, Ferney Diaz ${ }^{1}$, \\ Xiaoli Tang ${ }^{2}$, Lucille Lee ${ }^{1}$, Eric Klein ${ }^{1}$ \\ ${ }^{1}$ Department of Radiation Medicine, Northwell Health, New York, NY, USA \\ ${ }^{2}$ MSKCC, New York, NY, USA \\ Email:xqia n@northwell.edu
}

How to cite this paper: Qian, X., Vaidya, K., Puckett, L., Diaz, F., Tang, X.L., Lee, L. and Klein, E. (2017) A Post-Mastectomy Radiation Therapy Dose Distribution Study. International Journal of Medical Physics, Clinical Engineering and Radiation Oncology, 6, 457-467.

https://doi.org/10.4236/ijmpcero.2017.64041

Received: November 6, 2017

Accepted: November 26, 2017

Published: November 29, 2017

Copyright $\odot 2017$ by authors and Scientific Research Publishing Inc. This work is licensed under the Creative Commons Attribution International License (CC BY 4.0).

http://creativecommons.org/licenses/by/4.0/

\begin{abstract}
Purpose: For post-mastectomy radiation therapy, skin dose must be accurately estimated to assess skin reactions, such as: erythema, desquamation, and necrosis. Even with advanced algorithms, planning systems do not always provide accurate dosimetry for target volumes distal to skin. Methods and Materials: In this study, a female anthropomorphic (ART) phantom and the newest generation of optically stimulated luminescence dosimeters (OSLD) (nanoDots, Landauer Inc.) were deployed to measure chest wall dose distribution. Since actual dose to patients' lung and heart cannot be measured using in-vivo dosimetry, film was also used to verify the dose distribution to the left lung and heart. The treatment planning was performed using tolerance limits of $95 \%$ to $107 \%$ of prescription dose. The ART phantom was irradiated according to 3 three-dimensional (3D) conformal radiotherapy plans for $200 \mathrm{cGy}$ dose per fraction using $6 \mathrm{MV}$ medial and lateral tangential photon beams. The dose distribution provided by treatment planning was studied using nanoDots and film. Results: Results show that the largest surface dose difference between nanoDots measurement and prescribed dose for medial and lateral tangential beams, are $3.8 \%$ and $9.8 \%$, respectively. This difference may be due to higher effective point of measurement and angular dependence of the nanoDots. The maximum differences in measured dose compared with prescribed dose, using film for heart and the left lung, were $6.2 \%$ and $7.5 \%$ respectively. Conclusions: Both nanoDots and film provided reasonable estimation of dose distribution in post-mastectomy radiation therapy.
\end{abstract}

\section{Keywords}

Breast Radiotherapy, Post-Mastectomy, Chest-Wall Surface Dose, OSLD, NanoDot 


\section{Introduction}

Breast cancer is one of the most common cancers affecting women. Approximately 1 in 8 American women will develop breast cancer over the course of her lifetime [1]. Breast cancer mortality is second only to lung cancer. In order to minimize the risk of breast cancer recurrence, breast cancer patients are often treated using adjuvant radiation therapy after surgery [2]. However, the radiation therapy can cause adverse skin reactions, such as painful acute desquamation and chronic fibrosis [3]. Skin reaction tends to be most severe for postmastectomy chest wall cases treated using bolus. Therefore, the assessment of skin dose is important to evaluate the risk of side effects from radiation treatment. According to the International Commission on Radiological Protection (ICRP)'s Publication No.60 [4], the skin dose should be assessed for the dermis and epidermis layers of the skin. Although we are able to estimate dose to a patient using the radiation treatment planning system (TPS) [5] [6] [7], a number of studies have demonstrated that surface and near-surface doses estimated by TPS can be inaccurate [8] [9] [10]. One reason is the steep dose gradient in this region. The ultimate check of the actual dose delivered to a patient in radiotherapy can be achieved by using in-vivo dosimetry [9]. The in-vivo dosimetry is applied to assess the actual dose delivered to accessible critical organs such as rectum, vagina and bladder; or in difficult geometries organs where the dose is hard to predict from the treatment plan such as head-and-neck and breast cancers [11].

Many techniques have been used for in-vivo dosimetry, such as semiconductor diodes, thermoluminescence dosimeter, and metal oxide semiconductor field effect transistor (MOSFET) [12] [13] [14] [15]. In this study, the dosimetry estimate using nanoDots (Landauer Inc., Glenwood, Ill.) and Gafchromic EBT2 film (Ashland Inc., Wayne, NJ) was carried out to measure chest wall dose distribution for parallel opposed beams using treatment planning.

Cardiac toxicity is a well-established late effect of radiation therapy to the chest wall, such as coronary artery disease, which may occur years after radiation treatment. Lung toxicity is not as commonly reported, but irradiation of larger volumes can lead to radiation pneumonitis. Dose in heart and left lung should be closely monitored in post-mastectomy radiation therapy [16] [17] [18] [19] [20]. Certain organs are inaccessible for in vivo dosimetry, such as the heart and lung. Therefore, a phantom was used to measure delivered dose to these organs.

\section{Methods and Materials}

\subsection{NanoDot Calibration}

Compared with other types of optically stimulated luminescence dosimeters (OSLDs), the nanoDotis the most feasible dosimeter for single point measurements of skin dose assessment in radiotherapy application due to its small size. The detector material is aluminum oxide doped with carbon $\left(\mathrm{Al}_{2} \mathrm{O}_{3}: \mathrm{C}\right)$ with 1.2 $\mathrm{mm}$ thickness and $5 \mathrm{~mm}$ diameter encased in $10 \mathrm{~mm} \times 10 \mathrm{~mm} \times 1.8 \mathrm{~mm}$ light 
tight plastic holder, as shown in Figure 1. The plastic holder has a density of $1.03 \mathrm{~g} / \mathrm{cm}^{3}$, and the leaf thickness covering the front and back of the nanoDots is $0.36 \mathrm{~mm}[21]$.

Before using nanoDots for dose measurements, they were calibrated under full buildup conditions at $100 \mathrm{~cm} \mathrm{SAD} \mathrm{for} 6 \mathrm{MV}$ photons. The calibration was carried out using solid water slabs (Gammex Inc., Middleton, WI) and $0.5 \mathrm{~cm}$ bolus. The slabs consisted of various thickness slabs from 0.2 to $5 \mathrm{~cm}$ with dimensions of $30 \mathrm{~cm} \times 30 \mathrm{~cm}$ and having a density of $1.045 \mathrm{~g} / \mathrm{cm}^{3}$. The nanoDots were placed on $10 \mathrm{~cm}$ thick slabs, positioned on the central axis at depth of maximum dose, $1.5 \mathrm{~cm}$ for $6 \mathrm{MV}$ photons beam (Truebeam, Varian Inc., Palo Alto, CA). A half centimeter bolus and $1.0 \mathrm{~cm}$ solid water phantom were placed above the nanoDot. The linear accelerator was calibrated for a SAD of $100 \mathrm{~cm}$ and field size of $10 \mathrm{~cm} \times 10 \mathrm{~cm}$ for an output of $1 \mathrm{cGy} / \mathrm{MU}$. The nanoDots were irradiated for absorbed dose of 2 cGy, 5 cGy, 8 cGy, 50 cGy, 100 cGy, 150 cGy, 200 cGy and $300 \mathrm{cGy}$. One dosimeter was kept as a control for background measurement. The nanoDots were read using the microStarii reader (Landauer Inc., Glenwood, Ill.). Results were presented as a calibration curve of nanoDots reading value versus absorbed dose to water. A linear relationship of dose/reading for nanoDots was determined.

In addition, a study on the angular dependence of nanoDots was carried out. NanoDots were irradiated with $200 \mathrm{MU} / \mathrm{min}$ at gantry angle of $0^{\circ}, 30^{\circ}, 60^{\circ}, 90^{\circ}$, $270^{\circ}, 300^{\circ}$ and $330^{\circ}$, the setup is shown in Figure 2. Results are for relative dose versus gantry angle.

\subsection{Film Calibration}

The Gafchromic EBT2 film (Ashland Inc., Wayne, NJ) was calibrated under the same conditions as the nanoDot. The calibration consisted of 12 dose points spanning 0 to $400 \mathrm{cGy}$. Singular films for each dose level were positioned at the isocenter plane. Guides were marked on the slab to aid film placement. For improved accuracy of the EBT2 film calibration, each exposure was repeated twice. The final calibration was derived from the average response of the films.

After film calibration, the percentage depth dose (PDD) values were measured using films loaded on the slab stack at the following depths: 0 (surface), 1.5, 3, 7,

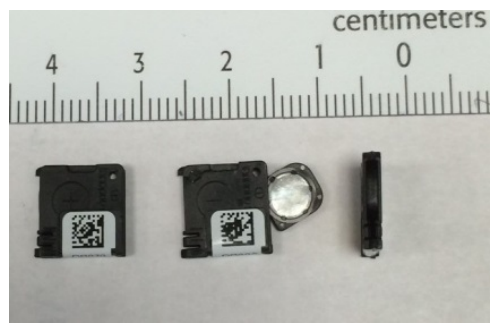

Figure 1. AnanoDot, material used is Aluminum oxide doped with Carbon $\left(\mathrm{Al}_{2} \mathrm{O}_{3}: \mathrm{C}\right)$ with $1.2 \mathrm{~mm}$ thickness and $5 \mathrm{~mm}$ diameter encased in $10 \mathrm{~mm} \times 10 \mathrm{~mm} \times 1.8 \mathrm{~mm}$ light tight plastic holder. 


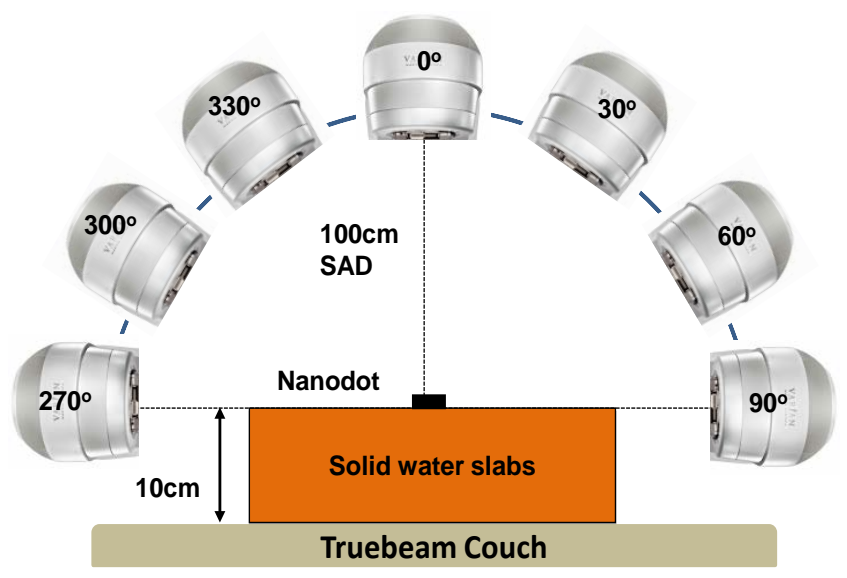

Figure 2. Schematic diagram of angular dependence on nanoDots. The gantry angles were at $30^{\circ}, 60^{\circ}, 90^{\circ}, 330^{\circ}, 300^{\circ}$ and $370^{\circ}$.

and $10 \mathrm{~cm}$. Films were orientated perpendicular to the radiation beam. Note that in reality these depths increase slightly when the finite thickness of the film (approximately $0.3 \mathrm{~mm}$ ) is accounted for. For comparison, PDD curves were also measured from films aligned vertically along the central beam axis, aligned using side lasers. Following the proper procedure suggested by the manufacture, each film was scanned with $300 \mathrm{dpi}$ using a film scanner (Epson expression $10000 \mathrm{XL}$, Long Beach, CA). The optical density was computed by subtracting the pre-scan from the post-scan [22] [23]. To determine the dose at the certain depth the polynomial fitting equation obtained from film calibration was employed to convert optical density to dose. The ImageJ (NIH, Bethesda, Maryland) software package was used to analyze the PDD.

\subsection{Treatment Planning Using an ART Phantom}

A female ART phantom was used in this study, as shown in Figure 3(a). The portion utilized in this work was the left chest wall. The phantom was constructed with a natural human skeleton cast inside soft tissue simulating material. Three tissue-simulating materials are the soft tissue material with a density of 0.997 $\mathrm{g} / \mathrm{cm}^{3}$, designed to have the same absorption as human tissue. The skeleton's bones possess a density of $1.610 \mathrm{~g} / \mathrm{cm}^{3}$, and lungs with a density of $0.330 \mathrm{~g} / \mathrm{cm}^{3}$. Film was placed between transverse slabs of ART phantom to measure dose to left lung and heart. Two layers of $0.5 \mathrm{~cm}$ thick bolus were used to cover the left chest. Four nanoDots were placed above the bolus, between the layers, and below the bolus, as shown in Figure 3(b) and Figure 3(c). Three control nanoDots were placed on chest wall to quantify imaging dose [24]. The phantom was scanned using CT simulator (Somatom, Siemens Medical Systems Inc., Malvern, PA) with both nanoDots and film in place. Three 3D conformal radiotherapy plans with 200 cGydose per fraction using $6 \mathrm{MV}$ photon medial and lateral tangential fields were performed using planning system (Pinnacle ${ }^{3}$ 9.10, Philips, Andover, MA). All dosimeters were contoured on CT and dose was extracted. The ART phantom was positioned on the treatment couch at the same set-up 


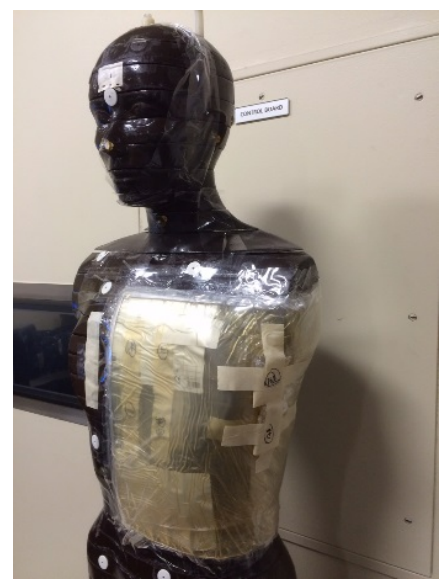

(a)

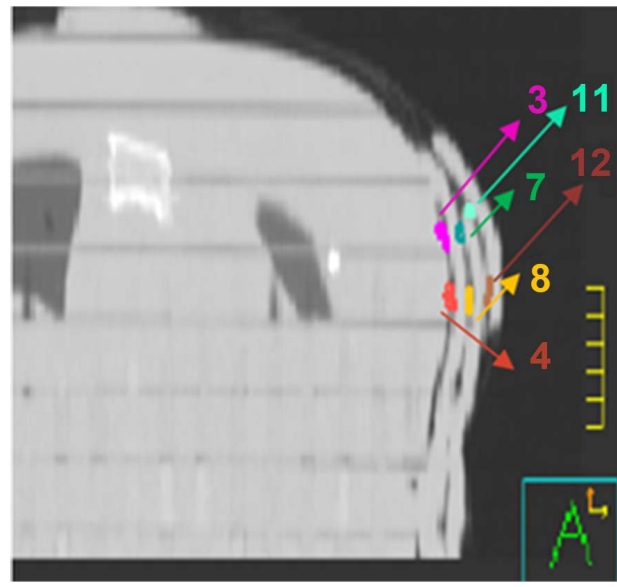

(b)

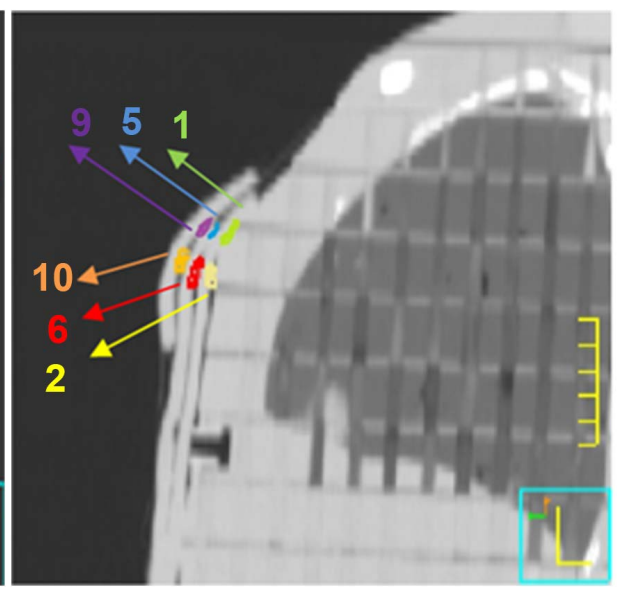

(c)

Figure 3. (a) Femal ART phantom; (b) Axial view of the scanned ART phantom; (c) Sagittal view. Positions of 12 nanoDots are labeled.

during the CT simulation, and irradiated with tangential fields (Truebeam, 6 MV, 200 cGy). The irradiation was performed three times using three sets of dosimeters for each plan. Irradiated nanoDots were read using microStarii reader. Film was scanned with a resolution of 300 dpi using Epson expression film scanner and analysed using DoseLab (Mobius, Houston, TA). The red channel data was used during film analysis, color correction was disabled as recommended by manufacture. All films were scanned approximately 24 hours after their irradiation.

\section{Results}

\subsection{Nanodot Calibration}

The nanoDot calibration curve for $6 \mathrm{MV}$ photons for the range of $0 \mathrm{cGy}$ to 300 cGy was established as shown in Figure 4. The graph shows that nanoDots signal is linearly proportional to absorbed dose to water. The linear equation obtained from the graph will be used in extrapolating the surface dose. Figure 5 shows the variation of surface dose for different angle of incident beam. The measured dose was normalized to the surface dose of perpendicular beam incidence. The curve is plotted with the least order polynomial. It shows that the incidence of beam entry angle on the phantom surface has an effect on surface dose; the more oblique the beam angle the higher the surface dose. This is possible due to the relative increase of thickness of nanoDot cover at oblique angles. The maximum angular response is given by $90^{\circ}$ gantry rotation. The application of angular correction factor is recommended for nanoDot when involving oblique radiation beams.

\subsection{Film Calibration}

A film calibration curve was obtained from films placed at isocenter plane and irradiated with $10 \times 10 \mathrm{~cm}^{2}$ field to doses within range of $0-400 \mathrm{cGy}$, as shown 


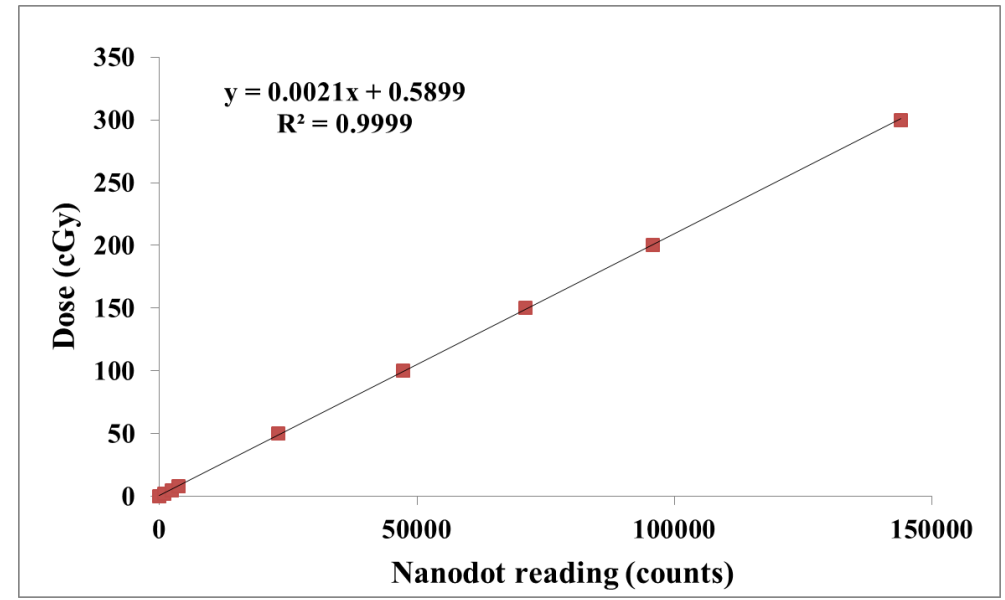

Figure 4. NanoDot calibration curve for $6 \mathrm{MV}$ photon beam.

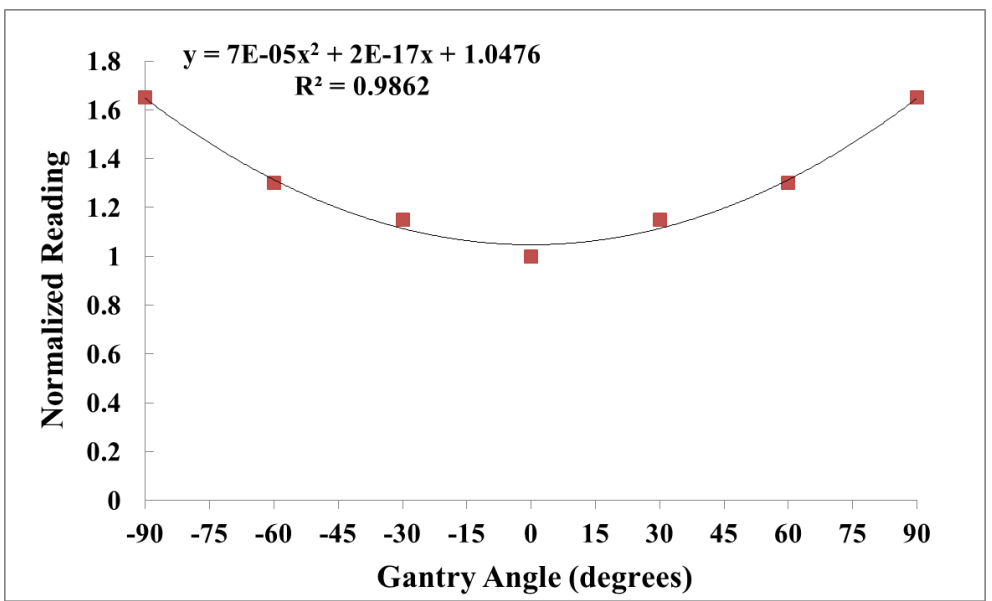

Figure 5. Response of nanoDot with the variation of beam direction.

in Figure 6(a). Figure 6(b) shows measured PDD for $10 \times 10 \mathrm{~cm}^{2}$ fields. The solid curve is PDD measured using EBT2 films vertically aligned with beam central axis. The markers indicate the PDD obtained from axial films at multi-depths.

\subsection{Dose Distribution to the Chest Wall}

The comparison of surface dose for parallel opposed treatment plans using nanoDots were made. In order to assess the reproducibility of the measurements, the surface dose for each plan was measured three times. In total, 36 nanoDots for each plan were evaluated in this study. The average dose for surface nanoDots at positions \#9 - 12 for plan 1 was: 197.2 cGy, $194.6 \mathrm{cGy}, 207.6 \mathrm{cGy}$ and $202.2 \mathrm{cGy}$, and the dose deviation compared with prescribed dose was: $3.8 \%$, 2.7\%, 9.8\% and 5.0\%, as shown in Figure 7. NanoDots at lateral positions, with greater beam obliquity, had larger variance than those at medial positions. A similar trend was observed for other nanoDots as shown in the Figure 7; \#5 - 8 were between the bolus layers, \#1 - 4 were below the bolus layers. We also noted 


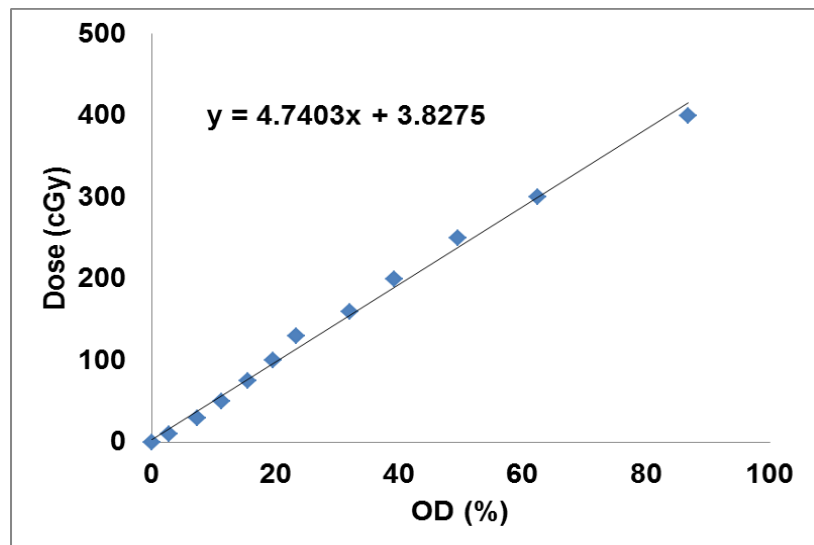

(a)

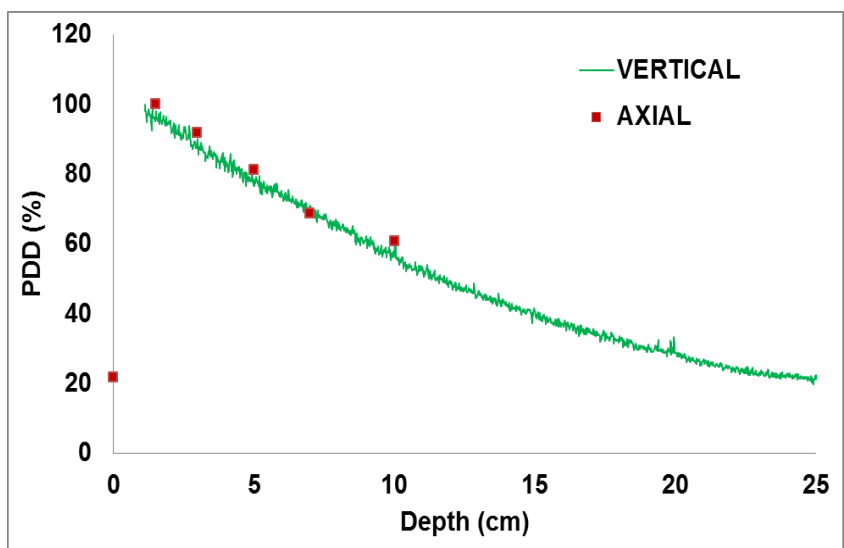

(b)

Figure 6. (a) Film calibration curve; (b) PDD comparison with film is vertical and axial to photon beam.

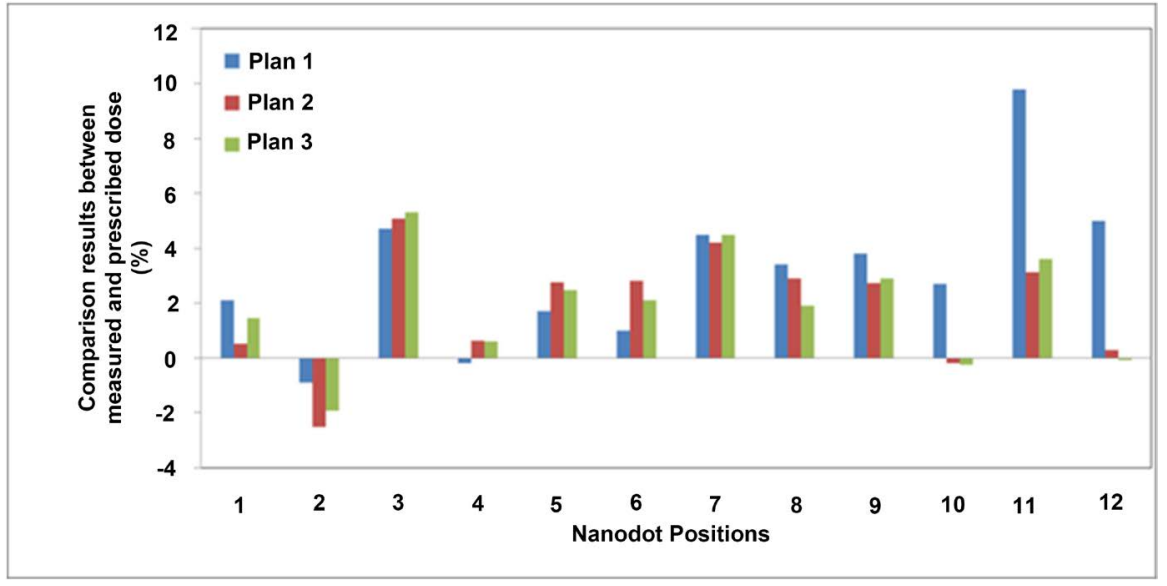

Figure 7. Comparison of measured and prescribed dose using nanoDots at different locations at chest wall, as shown in Figure 3.

that the surface dose at lateral tangential angle is higher than medial tangential angle after applying angle correction. This might be due to effect of exit dose, which may contribute to the surface dose, particularly for small separation body sections such as the chest wall. Thus, the surface dose that was measured was actually the combination of entrance and exit dose.

The measured dose using film for heart and the left lung was extracted along the red line drawn in Figure 8(c); a 6.2\% and 7.5\% maximum difference from prescribed dose was shown in Figure 8(d).

\section{Discussion}

In this study, we conducted measurements to describe the relationship between the prescribed dose and the measured dose to chest wall, left lung and heart. The dose distribution study using nanoDots and film was investigated using an ART phantom. Three parallel-opposed 3D conformal radiotherapy treatments were planned and delivered onto the ART phantom. The surface dose for the lateral 


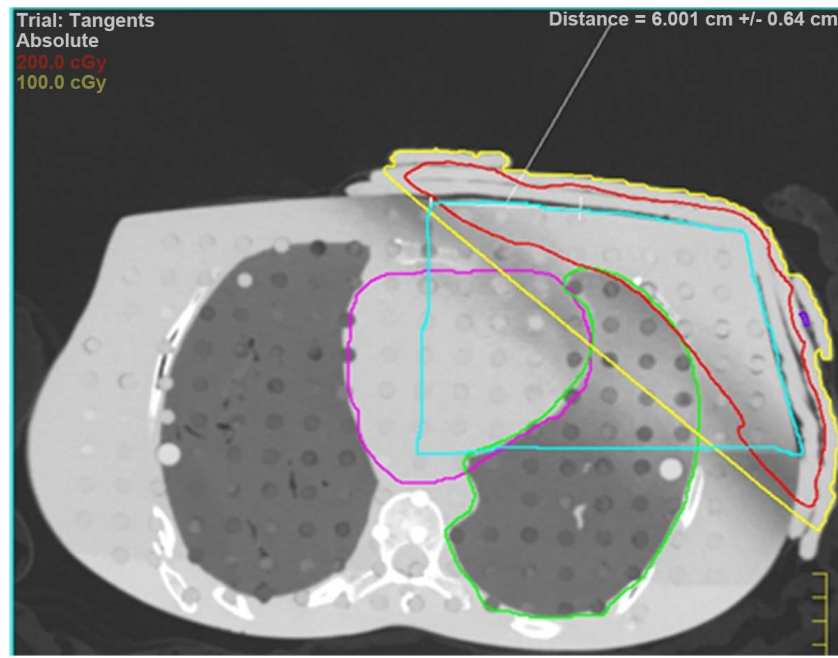

(a)

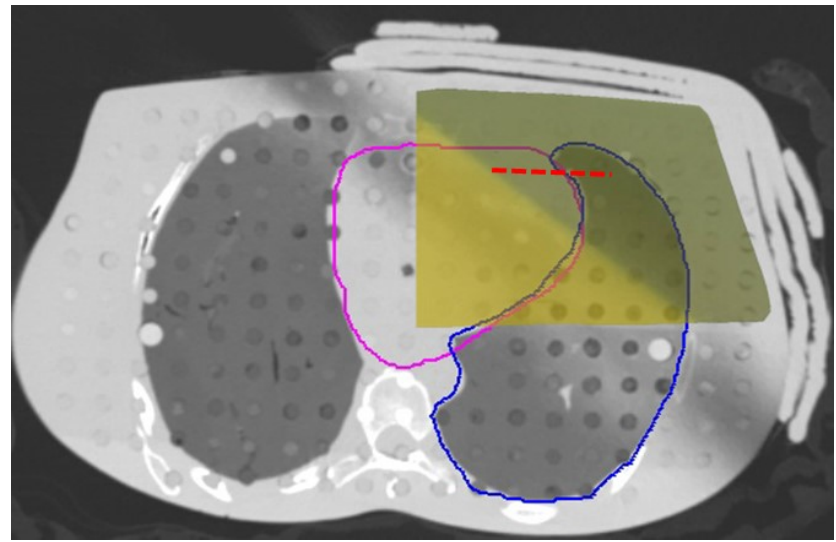

(c)

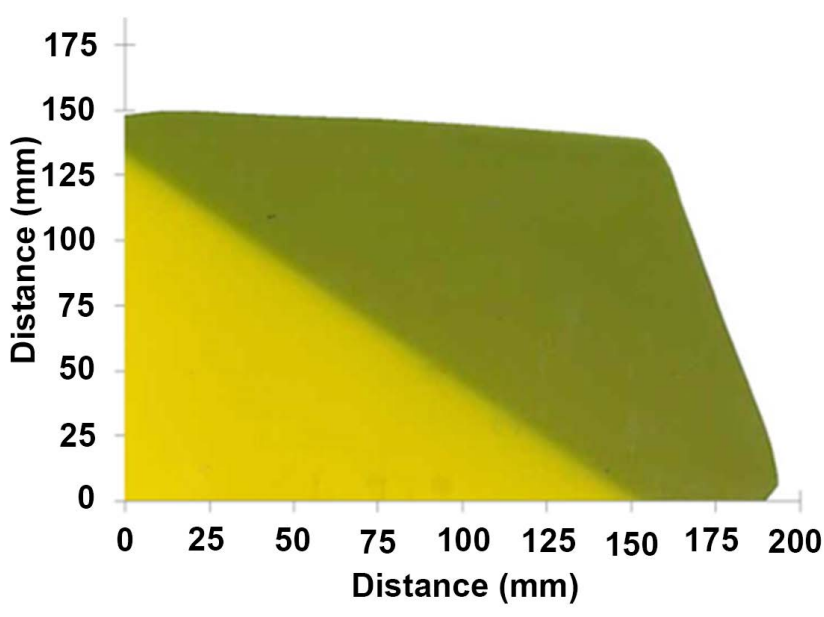

(b)

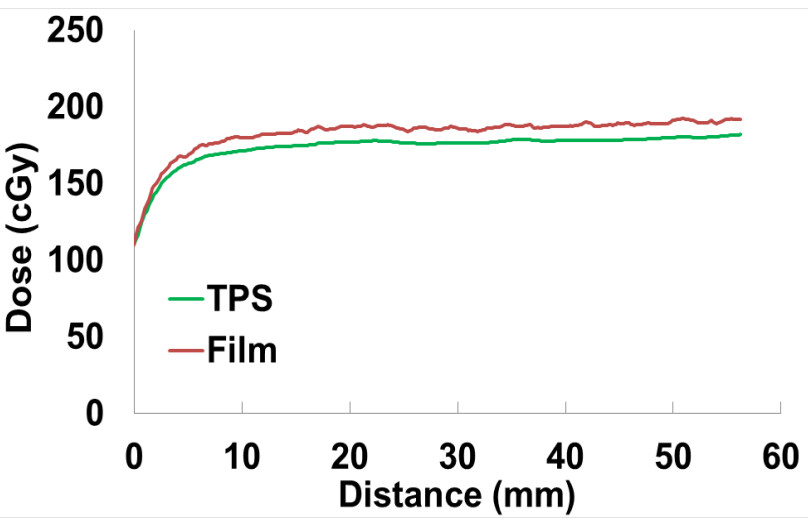

(d)

Figure 8. (a) Plane from planning CT, structure highlighted in blue is the embedded film; (b) Irradiated film was scanned; (c) Registration between Figure 8(a) and Figure 8(b). The RED dashed line shows the position where dose in left lung and heart was extracted; (d) Dose profile in heart and left lung along the RED dashed line drawn in Figure 8(c).

tangential field resulted in a higher measured dose than medial tangential field due to the effect of the radiation beam angle incidence and opposed beam exit dose.

Based on the results of this study, if nanoDots are used to assess for multi-field treatment, an angular dependence correction factor must be in place.

There are certain limitations in this preliminary study. First, we did not perform dose comparison for IMRT plans [25] [26] [27]. Second, beyond the advantageous characteristics of nanoDots as mentioned above, there are a number of other characteristics that must be accounted for when measuring, such as temperature, energy dependence, and linear energy transfer dependence [28] [29] [30] [31]. Last, plans using electron beams, which are commonly used to treat the chest wall, were not performed. It should be noted that for CT X-ray energies, the response of nanoDot is increased significantly [24] [30]. This is due to the relatively high $\mathrm{Z}$ value of $\mathrm{Al}_{2} \mathrm{O}_{3}$, prompting more photoelectric photon interactions. Three 
control nanoDots were used when ART phantom was scanned using a CT simulator.

\section{Conclusion}

The dosimetry provided by an advanced treatment planning system was verified using nanoDots and film. Both nanoDots and film provided reasonable estimation of dose distribution in post-mastectomy radiation therapy. Although surface dose measured using nanoDots does not give an exact estimate of skin dose due to the finite size of the detector, it is a useful method for in-vivo estimation of skin dose. There is no established method for accurately quantifying treatment-associated coronary artery and lung diseases, which may occur years after radiation treatment. Dose distribution estimate in heart and lung using film and ART phantom is a useful tool in the evaluation of breast cancer patients after treatment.

\section{References}

[1] http://www.breastcancer.org/symptoms/understand_bc/statistics

[2] Anderson, K.E., Mongin, S.J., Sinha, R., et al. (2012) Pancreatic Cancer Risk: Associations with Meat-Derived Carcinogen Intake in the Prostate, Lung, Colorectal, and Ovarian Cancer Screening Trial (PLCO) Cohort. Molecular Carcinogenesis, 51, 128-137. https://doi.org/10.1002/mc.20794

[3] Panettierei, V., Barsoum, P., Westermark, M., Brualla, L. and Lax, I. (2009) AAA and PBC Calculation Accuracy in the Surface Build-Up Region in Tangential Beam Treatments. Phantom and Breast Case Study with the Monte Carlo Code PENELOPE. Radiotherapy \& Oncology, 93, 94-101. https://doi.org/10.1016/j.radonc.2009.05.010

[4] International Commission on Radiological Protection (ICRP) (1991) ICRP Publication 60. Pergamon, Oxford.

[5] Costa, A.M., Barbi, G.L., Bertucci, E.C., Ferreira, H., Sansavino, S.Z., Colenci, B. and Caldas, L.V. (2010) In-Vivo Dosimetry with Thermoluminescent Dosimeters in External Photon Beam Radiotherapy. Applied Radiation and Isotopes, 68, 760-762. https://doi.org/10.1016/j.apradiso.2009.09.039

[6] Kry, S.F., Smith, S.A., Weathers, R. and Stovall, M. (2012) Skin Dose during Radiotherapy: A Summary and General Estimation Technique. Journal of Applied Clinical Medical Physics, 13, 20-34. https://doi.org/10.1120/jacmp.v13i3.3734

[7] Nakano, M., Hill, R.F., Whitaker, M., Kim, J.H. and Kuncic, Z. (2012) A Study of Surface Dosimetry for Breast Cancer Radiotherapy Treatments Using Gafchromic EBT2 Film. Journal of Applied Clinical Medical Physics, 13, 3727. https://doi.org/10.1120/jacmp.v13i3.3727

[8] Abella, V., Miro, R., Juste, B. and Verdu, G. (2011) Comparison of MCNP5 Dose Calculations inside the RANDO ${ }^{\circledR}$ Phantom Irradiated with a MLC LinAc Photon Beam against Treatment Planning System PLUNC. Progress in Nuclear Science and Technology, 2, 232-236. https://doi.org/10.15669/pnst.2.232

[9] Nilsson, B. and Sorcini, B. (1989) Surface Dose Measurements in Clinical Photon Beams. Acta Oncologica, 28, 537-542. https://doi.org/10.3109/02841868909092265

[10] International Atomic Energy Agency (IAEA) (2000) Absorbed Dose Determination in External Beam Radiotherapy: An International Code of Practice for Dosimetry 
Based on Standards of Absorbed Dose to Water, Technical Reports Series No. 398. IAEA, Washington DC.

[11] Lee, N., Chuang, C., Quivey, J.M., et al. (2002) Skin Toxicity Due to Intensity-Modulated Radiotherapy for Head-and-Neck Carcinoma. International Journal of Radiation Oncology, Biology, Physics, 53, 630-637. https://doi.org/10.1016/S0360-3016(02)02756-6

[12] Kron, T., Elliot, A., Wong, T., Showell, G., Clubb, B. and Metcalfe, P. (1993) X-Ray Surface Dose Measurements Using TLD Extrapolation. Medical Physics, 20, 703-711. https://doi.org/10.1118/1.597019

[13] Metcalfe, P., Hoban, P. and Kron, T. (1997) The Physics of Radiotherapy X-Rays from Linear Accelerators. Medical Physics Publishing, Madison.

[14] Rapley, P. (2006) Surface Dose Measurement Using TLD Powder Extrapolation. Medical Dosimetry, 31, 209-215. https://doi.org/10.1016/j.meddos.2006.02.003

[15] Dogan, N. and Glasgow, G.P. (2003) Surface and Build-Up Region Dosimetry for Obliquely Incident Intensity Modulated Radiotherapy 6 MV X-Ray. Medical Physics, 30, 3091-3096. https://doi.org/10.1118/1.1625116

[16] Wennberg, B., Gagliardi, G., Sundbom, L., Svane, G. and Lind, P. (2002) Early Response of Lung in Breast Cancer Irradiation: Radiologic Density Changes Measured by CT and Symptomatic Radiation Pneumonitis. International Journal of Radiation Oncology, Biology, Physics, 52, 1196-1206. https://doi.org/10.1016/S0360-3016(01)02770-5

[17] Kahan, Z., Csenki, M., Varga, Z., Szil, E., Cserhati, A., Balogh, A., Gyulai, Z., Mandi, Y., Boda, K. and Thurzo, L. (2007) The risk of early and late lung sequelae after conformal radiotherapy in breast cancer patients. International Journal of Radiation Oncology, Biology, Physics, 68, 673-681. https://doi.org/10.1016/j.ijrobp.2006.12.016

[18] Agatston, A.S., Janowitz, W.R., Hildner, F.J., Zusmer, N.R., Viamonte, M. and Detrano, R. (1990) Quantification of Coronary Artery Calcium Using Ultrafast Computed Tomography. Journal of the American College of Cardiology, 15, 827-832. https://doi.org/10.1016/0735-1097(90)90282-T

[19] Orban de Xivry, J., Janssens, G., Bosmans, G., et al. (2007) Tumour Delineation and Cumulative Dose Computation in Radiotherapy Based on Deformable Registration of Respiratory Correlated CT Images of Lung Cancer Patients. Radiotherapy \& Oncology, 85, 232-238. https://doi.org/10.1016/j.radonc.2007.08.012

[20] Feng, M., Moran, J.M., Koelling, T., et al. (2011) Development and Validation of a Heart Atlas to Study Cardiac Exposure to Radiation Following Treatment for Breast Cancer. International Journal of Radiation Oncology, Biology, Physics, 79, 10-18. https://doi.org/10.1016/j.ijrobp.2009.10.058

[21] Jursinic, P.A. (2010) Changes in Optically Stimulated Luminescent Dosimeter (OSLD) Dosimetric Characteristics with Accumulated Dose. Medical Physics, 37, 132-140. https://doi.org/10.1118/1.3267489

[22] Devic, S., Wang, Z., Tomic, N. and Podgorsak, E.B. (2006) Sensitivity of Linear CCD Array Based Film Scanners Used for Film Dosimetry. Medical Physics, 33 3993-3996. https://doi.org/10.1118/1.2357836

[23] Saur, S. and Frengen, J. (2008) GafChromic EBT Film Dosimetry with Flatbed CCD Scanner: A Novel Background Correction Method and Full Dose Uncertainty Analysis. Medical Physics, 35, 3094-3101. https://doi.org/10.1118/1.2938522

[24] Al-Senan, R.M. and Hatab, M.R. (2011) Characteristics of an OSLD in the Diagnostic Energy Range. Medical Physics, 38, 4396-4405. 
https://doi.org/10.1118/1.3602456

[25] Rudat, V., Alaradi, A.A., Mohamed, A., Ai-Yahya, K. and Altuwaijri, S. (2011) Tangential Beam IMRT versus Tangential Beam 3D-CRT of the Chest Wall in Postmastectomy Breast Cancer Patients: A Dosimetric Comparison. Radiation Oncology, 6, 26. https://doi.org/10.1186/1748-717X-6-26

[26] Krueger, E.A., Fraass, B.A., McShan, D.L., Marsh, R. and Pierce, L.J. (2003) Potential Gains for Irradiation of Chest Wall and Regional Nodes with Intensity Modulated Radiotherapy. International Journal of Radiation Oncology ${ }^{*}$ Biology ${ }^{*}$ Physics, 56, 1023-1037. https://doi.org/10.1016/S0360-3016(03)00183-4

[27] van der Laan, H.P., Korevaar, E.W., Dolsma, W.V., Maduro, J.H. and Langendijk, J.A. (2010) Minimising Contralateral Breast Dose in Post-Mastectomy Intensity-Modulated Radiotherapy by Incorporating Conformal Electron Irradiation. Radiotherapy \& Oncology, 94, 235-240.

https://doi.org/10.1016/j.radonc.2009.12.015

[28] Quach, K.Y., Morales, J., Butson, M.J., Rosenfeld, A.B. and Metcalfe, P.E. (2000) Measurement of Radiotherapy X-Ray Skin Dose on a Chest Wall Phantom. Medical Physics, 27, 1676-1680. https://doi.org/10.1118/1.599035

[29] Kerns, J.R., Kry, S.F., Sahoo, N., Followill, D.S. and Ibbott, G.S. (2011) Angular Dependence of the nanoDot OSL Dosimeter. Medical Physics, 38, 3955-3962. https://doi.org/10.1118/1.3596533

[30] Jursinic, P.A. (2007) Characterization of Optically Stimulated Luminescent Dosimeters, OSLDs, for Clinical Dosimetric Measurements. Medical Physics, 34, 4594-4604. https://doi.org/10.1118/1.2804555

[31] Yukihara, E.G., Mardirossian, G., Mirzasadeghi, M., Guduru, S. and Ahmad, S. (2008) Evaluation of $\mathrm{Al}_{2} \mathrm{O}_{3}$ :C Optically Stimulated Luminescence (OSL) Dosimeters for Passive Dosimetry of High-Energy Photon and Electron Beams in Radiotherapy. Medical Physics, 35, 260-269. https://doi.org/10.1118/1.2816106 